\title{
IL CARTEGGIO GOR'KIJ-OSORGIN: LE LETTERE “ITALIANE” (1910-1913)
}

\author{
ANASTASIA PASQUINELLI
}

ABSTRACT. - Nell'intervento viene commentata la prima parte del carteggio tra Gor'kij e Osorgin svoltosi tra il '10 e il '13: si tratta appunto delle sette lettere "italiane", relative al periodo trascorso allora da entrambi in Italia: le rimanenti 49 lettere (1924-1936), sono scambiate tra Francia - per Osorgin -, Italia e URSS - per Gor'kij. Il pregio particolare di questo copioso carteggio - riordinato e pubblicato nel 2002 - sta nel fatto di essere stata l'unica corrispondenza di Gor'kij tanto prolungata e costante con uno scrittore dell'emigrazione.

Infatti, nonostante una netta incompatibilità politica, tra momenti polemici, segrete speranze e sgradevoli malintesi, tra i due corrispondenti si stabilisce una sintonia destinata a durare a lungo. Il doloroso problema della triste situazione degli emigrati russi appare fondamentale in queste lettere, alla cui base stanno problemi politici, personali ed artistici, specialmente nel difficile momento dell'amnistia zarista del 1913, da cui Osorgin era escluso, mentre Gor'kij esitava ad accettarla. All'atteggiamento incerto di Gor'kij si contrappone allora la visione radicale di Osorgin.

$* * *$

В выступлении комментируется первая часть переписки; речь идет о семи письмах относящихся к периоду современно обоими писателями проведенному в Италии. Остальные 49 письма 1924-1936 обменялись между Францией Осоргин, Италией и СССР Горький.

Особый интерес переписки в том, что несмотря на политическую несовместимость, на полемическое часто настроение корреспондентов, она явилась единственной столь длительной и постоянной горьковской перепиской с писателем, находившийся в эмиграции.

В «итальянских» письмах стоит главная тема трудной жизни тогдашней русской эмиграции, кроме личных и творческих отношений двух писателей, особенно в трудных днях царской амнистии 1913, налагающей на эмигрантов тяжелые решения 
Il testo da me consultato è una raccolta - sbornik - ad opera di vari studiosi, delle corrispondenze finora inedite tra scrittori russi (tranne una fra Thomas Mann e Bunin) residenti in Russia gli uni, emigrati all'estero gli altri. ${ }^{1}$ La corrispondenza reperita fra Gor'kij e Osorgin è a cura di M. A. Bočarova, già nota studiosa del primo scrittore.

"Il pregio particolare di questa corrispondenza ora riordinata e pubblicata (ancorche' incompleta), sottolinea la curatrice - sta nel fatto di esser stata l'unica corrispondenza di Gor'kij tanto prolungata e costante con uno scrittore dell'emigrazione". ${ }^{2}$

Il materiale epistolare - suddiviso cronologicamente in due parti disuguali - abbraccia circa un quarto di secolo, con un totale - nel periodo tra il 1910 ed il '13, di sette lettere, 4 di Osorgin e 3 di Gor'kij; la seconda parte, tra il '24 e il '36, conta 5 lettere di Gor'kij contro le 44 di Osorgin, cioè 55 lettere in tutto. Questo perché le lettere di Gor'kij a Osorgin andarono perse e poi probabilmente distrutte durante l'occupazione tedesca, quando Osorgin e la moglie, abbandonata la loro casa parigina, si rifugiarono nel paesino di Chabris, dove lo scrittore si spense nel novembre $1942^{3}$

Mi occuperò oggi della prima parte di questa corrispondenza - le 7 "lettere italiane" - perché una trattazione completa non si può svolgere in questa sede senza togliere a questo significativo corpus il suo particolare interesse storico, politico, letterario ed umano.

Sembra che il primo incontro tra i due corrispondenti sia avvenuto nei primi mesi del 1903 nell'appartamento di Marija Andreevna Astrova, dove Osorgin si era recato per un incontro del Comitato per l'assistenza ai poveri, del quale anch'egli era membro. In un articolo del $1934,{ }^{4}$ Osorgin rievoca l'emozione allora vivamente provata nello scor-

1. AA. VV. (P. Davis, V. Keldys, a cura di), $S$ dvuch beregov. Russkaja literatura XX veka v Rossii i za rubežom, Mosca, IMLI, 2002, pp.823; in particolare, la seconda parte del volume - cinque studi - riguarda gli scambi epistolari tra Gor'kij ed altri scrittori emigrati.

2. I. A. Bočarova, M. Gor'kij i M. Osorgin. Perepiska, in op. cit., pp. 387-539. La citazione è a p. 387.

3. Per lettere di Osorgin a Gor'kij e le loro complicate vicende archivistiche russe, rimandiamo allo studio di I. A. Bocarova, op. cit., Perepiska..., p. 387. Inoltre, N. N. Berberova, Železnaja ženščina (La donna di ferro), New York, 1981, pp 259 sgg., dedicate al problema degli archivi personali di Osorgin. Cfr. anche A. Becca Pasquinelli, La vita e le opinioni di M. A. Osorgin (1878-1942), Firenze, 1986, pp. 150-152.

4. M. Osorgin, Julija Michajlovna Astrova, in Poslednie Novosti, 3 aprile 
gere l'ormai famoso scrittore: "Mi pareva - scrive Osorgin - di assistere ad una scena storica, di cui sarebbe rimasto ai posteri il ricordo".

In questi personaggi, nati a un decennio di distanza l'uno dall'altro (nel 1868 Gor'kij, Osorgin nel 1878) ravvisiamo due diverse figure tipiche di quella società russa in fermento, avvicinate dalle tempestose cruciali vicende del primo Novecento. Peškov, nato povero, presto orfano, vissuto per quasi trent'anni reietto - ricorda il giovane Nekrasov dei primi anni 1840? - vagabondo nei bassifondi urbani, suicida mancato, ribelle preso di mira e incarcerato dall'occhiuta polizia zarista, retto da grandi passioni e da una sagace pazienza, padrone dello "slog", premiato infine da una luminosa fortuna editoriale che coronava la sua tenace ambizione e dal carisma di una chiara luce intellettuale che commoveva mezzo mondo, non era infine la trionfale presenza di un nuovo raznočinec, nella tradizione che si ripeteva e si rinnovava in uno straordinario - scandaloso (come sospettava persino lo zar) caso culturale e politico globale?

Se Gor'kij era il proletario di genio, Osorgin era l'attivo neo-giornalista di famiglia borghese liberale di provincia: il padre magistrato, la madre diplomata; laureatosi in legge nel 1902, egli avrebbe fatto parte della gioventù di tendenze radicali, entrando nell'area dei social-rivoluzionari di matrice populista: tipico esempio, il giovane Osorgin, della cosiddetta nuova intelligencija delle professioni, con un occhio all'attività professionale e l'altro alla partecipazione politica, con i progetti ed i rischi inerenti.

Negli anni cruciali tra il 1905 e il 1907 le scelte politiche condizionano le loro drammatiche vicende personali: i loro percorsi di vita paralleli saranno successivamente intrecciati da incontri, come anche da una corrispondenza ricca di riflessioni e di progetti. Gor'kij, arrestato e imprigionato all'indomani della domenica di sangue del gennaio 1905 e liberato su cauzione un mese dopo, grazie al movimento di solidarietà europeo, dove si distingue l'intervento italiano, lascia la Russia nel gennaio 1906, con prima sosta a Helsinki, per un malriuscito viaggio negli USA. ${ }^{5}$ Nell'ottobre successivo sbarca a Napoli; dal 4 novembre risiede

1934, n . 4758; ripubblicato in M.O., Vospominanija. Povest' o sestre, a cura di O. G. Lasunskij, Voronež, 1992, pp. 78-82.

5. Il viaggio fu suggerito - sembra - da Leonid Krasin, allora consigliere finanziario del partito bolscevico con l'intento che Gor'kij raccogliesse fondi per il movimento 
a Capri, ritornando in patria il 31 dicembre 1913, garantito dalla famosa amnistia politica promulgata dallo zar il 21 febbraio di quell'anno, nella ricorrenza del trecentesimo anniversario della dinastia dei Romanov. La prospettiva del ritorno in Russia, dapprima accolta da Gor'kij con diffidenza, veniva accettata - in ritardo e con riluttanza - in seguito alle energiche rimostranze di Lenin, già intervenuto nel 1908 per distoglierlo dalle fumose divagazioni di bogostroitel'stvo ${ }^{6}$ cioè da una pericolosa deriva religioso/idealistica che finì col perderlo nella soggezione a Stalin di cui fu ostaggio e infine vittima.

A Osorgin, tra la fine del 1905 e quella del 1906 càpita più o meno lo stesso. Arrestato la notte del 18 dicembre 1905, all'indomani della rivolta moscovita della Presnja Rossa, e del sostanziale fallimento del movimento, trascorreva alcuni mesi nella prigione della Taganka. Liberato su cauzione nel maggio 1906, si rifugiava con un gruppo di compagni a Helsinki; ${ }^{7}$ nel dicembre 1906, dopo un viaggio attraverso l'Europa, giungeva a Sori, sulla Riviera ligure di Levante. Iniziava così per lui quell'esilio italiano che sarebbe durato fino al maggio 1916, quando egli avrebbe deciso, pur con rincrescimento, di lasciare il nostro Paese per far ritorno in Russia. Lasciata la Riviera nell'ottobre 1908, Osorgin si stabiliva a Roma, avviando una regolare collaborazione presso i due organi di stampa russi dell'epoca, Russkie Védomosti e Vestnik Evropy; tra il 1908 e il '13, nella veste "ufficiale" di corrispondente dall'Italia per questi due grandi giornali, egli ebbe anche certamente modo di incontrarsi a Roma e a Capri - dove accompagnava i gruppi di maestri russi nei loro periodici viaggi di studio - con il più famoso esule russo d'Italia. ${ }^{8}$

Non gli sfuggiva quindi la notizia "sensazionale" giuntagli con un "impreciso telegramma da Parigi" e rilevata dalla stampa italiana, in particolare dall' “Avanti!", quella nientemeno che dell'espulsione di

rivoluzionario. Il soggiorno di Gor'kij negli USA fu però un insuccesso. Cfr. a questo proposito M. Slonim, From Chekhov to the Revolution, Oxford Univ. Press, 1962, pp. 130-131.

6. Per la storia e l'ambiente di questo movimento, cfr. nella preziosa opera di A. Tamborra, Esuli russi in Italia dal 1905 al 1917, Bari, 1977, cap. XI, intitolato Lenin e il raskol ideologico a Capri e a Bologna; inoltre in P. Cazzola, L'Italia dei Russi tra Settecento e Novecento, vol II, ed. CIRVI, Moncalieri, 2004, p. 265, nel denso, interessante capitolo Artisti e scrittori russi a Capri. L'esilio politico di M. Gor'kij.

7. Cfr. A. Becca Pasquinelli, La vita..., op. cit., pp. 16-18.

8. Cfr. M.Osorgin, Un russo in Italia, a cura di A. Pasquinelli, Torino, 1997, pp. $51-118$ 
Gor'kij dal "seno del partito socialdemocratico russo" decisa nel novembre 1909. A questo pur gravissimo, ma poco noto episodio della biografia politica di Gor'kij, Osorgin dedicava un articolo forte, Gli italiani a proposito della "deposizione" di Gor'kij," decisamente in difesa dell'illustre scrittore, soprattutto in ordine alle "strane motivazioni" addotte dal partito, quale quella dello "stile di vita personale" di Gor'kij, citando appunto le righe indignate dell'"Avanti!" ("organo ufficiale del partito socialista italiano") sui pretesti per l'espulsione, nonché quelle del "Secolo" di Napoli, per il quale "l'autentico motivo era la divergenza ideologica dal vangelo del partito e dal dogma del partito". Un "caso" tipicamente russo diventava, con questo articolo, anche un "caso" italiano di cui Osorgin valutava, nell'interesse dei due Paesi, gli sviluppi, certamente nella prospettiva di una futura - ma non poi molto lontana - alleanza.

L'articolo di Osorgin non è una lettera, ma è certamente un appassionato messaggio di simpatia personale; la solidarietà dimostrata da Osorgin al grande "spodestato" consolidò in qualche modo un certo forse già latente - interesse da parte di Gor'kij al quale paradossalmente, dopo il nizloženie zarista dall'Accademia delle lettere nel 1902, toccava quello del 1909 dal partito SD.

Il fatto è che il nizloženie - come significativo "richiamo all'ordine" alla "scuola di Capri", dove Gor'kij era docente di letteratura russa - avvenne nella prima metà del novembre 1909: sembra quindi che Lenin stesso abbia voluto punire Gor'kij (già una volta ripreso per la sua pericolosa tendenza al bogostroitel'stvo), isolandolo e togliendogli così l'appoggio di una forte struttura (vangelo, dogma) alla quale potersi appoggiare, in un momento molto delicato delle relazioni russoitaliane; insospettisce soprattutto la coincidenza della misura restrittiva, dichiarata all'indomani - letteralmente - del famoso incontro di Racconigi avvenuto il 24 ottobre 1909 tra lo zar e il re d'Italia, concluso con un patto segreto di solidarietà, che avrebbe, di lì a pochi anni, ribaltato le alleanze delle cosiddette Triplici. Che cosa si temeva da Gor'kij? Cosa si voleva da lui? Che mossa (scritta) poteva egli avere in mente alla luce di Racconigi? Insieme alla "macchia bianca" di Gor'kij potrebbe essercene una nera dei SD? Comunque, le ipotesi menzionate sia dall'"Avanti!" che dal "Secolo" risultano entrambe sostanzialmente va-

9. M. Osorgin, Ital'jancy o nizloženii Gor'kogo, R.V., 27 novembre 1909, n. 272. 
ghe e inattendibili.

Ma Osorgin, osservatore così attento della vita politica italo-russa, aveva qualcosa in mente a questo proposito, e lo leggiamo nelle poche righe della prima lettera del carteggio qui in questione, che porta la data del 1 settembre 1910 (quasi un anno dopo il suddetto sbalorditivo episodio), come in quelle della breve risposta dello stesso Gorkij (n. ${ }^{\circ} 2$ ) a stretto giro di posta.

Nella suddetta lettera n. 1 del settembre 1910, Osorgin (su carta intestata ad uno dei giornali cui collaborava in qualità di "corrispondente speciale", RV), pone senz'altro a Gor'kij una domanda piuttosto indiscreta, addirittura impertinente: gli chiede di confermare o di smentire una notizia apparsa dapprima su un giornale francese e ripresa da alcuni giornali russi (di cui allega il ritaglio), tale che "Voi non avete potuto non notarla", e lo prega di fargli avere comunque "due parole" di risposta. Gli comunica premurosamente il proprio indirizzo presso "Russkie Vedomosti", assicurando in poscritto la propria discrezione, e la promessa di non divulgare la notizia nella stampa se non dietro espresso consenso del destinatario; un modello di comportamento giornalistico, ma anche un rischio professionale, del quale Osorgin aveva certamente valutato la portata.

Si trattava del trafiletto, comparso sull'Humanité del settembre 1910 (giornale diretto dal socialista J. Jaurès) ad opera di Amilcare Cipriani, il rivoluzionario anarchico italiano dalla vita avventurosa, sostenitore di manifestazioni anticlericali e soprattutto antimonarchiche, esule allora a Parigi: in tale scritto, intitolato Un Roi Socialiste, si comunicava che Gor'kij, durante il suo viaggio a Napoli del 5-7 agosto precedente, si era incontrato col re d'Italia Vittorio Emanuele III. ${ }^{10} \mathrm{La}$ risposta di Gor'kij (n. 2) non si fa attendere: senza menzionare direttamente il tipo della domanda fattagli, smentisce seccamente: "Ho già detto a suo tempo - scrive - che la notizia è errata". Rincara la dose, rinviando la domanda al mittente: "L'autore di questa comunicazione prosegue - è un certo signor Cipriani; lui solo può rispondere alla Vostra domanda su quel che gli è servito da 'motivo per il presente trafiletto"”; insomma, la provocazione di Osorgin veniva respinta al mit-

10. Sui giornali russi cui probabilmente Osorgin - come suggerisce Bočarova, $o p$. cit., p.488 - aveva inviato il ritaglio dell'Humanité -, questo fatto non accertato veniva interpretato come un "civettare del re coi socialisti". 
tente. La notizia dell'incontro napoletano di Vittorio Emanuele III, con Gor'kij in libera uscita, da poco "spodestato" dai SD, al tempo del governo Luzzatti - simpatico, tra l'altro, a Osorgin ${ }^{11}$ - e nel fermento delle manovre diplomatiche italo-russe inaugurate alla grande con Racconigi, sembra dunque restare per ora un enigma.

A spingere Osorgin alla forzatura epistolare su Gor'kij (sostenuta dal trafiletto a firma di Cipriani) potrebbe anche essere stata in buona misura la tendenza alla sovrapposizione ideologica tra due aree, quella social-rivoluzionaria russa e quella anarchica italiana, tipica degli scenari politici italo-russi dei primi anni del ' 900 , riassunta nelle parole che Osorgin riporta - pronunciate da un amico russo terrorista, impiccato a Mosca nel febbraio 1908: "In Italia io sono un anarchico, in Russia per ora posso essere un social-rivoluzionario". ${ }^{12}$ In questo caso, la lettera di Osorgin avrebbe forse addirittura mirato a indurre in tentazione il tetragono Gor'kij?

Le prime due lettere pubblicate aprono già un interessante orizzonte sullo scambio della successiva corrispondenza. La terza breve lettera pubblicata, che rompe un silenzio epistolare di quasi tre anni, molto probabilmente interrotti da brevi incontri personali, è firmata da M. Il'in (Osorgin), in data 2 marzo 1913, appena diffusa la notizia dell'amnistia zarista proclamata appunto il 21 febbraio di quell'anno.

In qualità di corrispondente di RV, Osorgin aveva deciso di incontrare subito Gor'kij, per raccogliere, cominciando da questo scrittore "dall'enorme fama letteraria", una serie di interviste ad altri eminenti emigranti russi sul tema della recente amnistia (anche per coloro che avevano lasciato la Russia dopo il 1905-1906), ma da cui egli stesso era rimasto escluso.

11. Cfr. A. Becca Pasquinelli, La vita..., op.cit., p. 51: " "(Osorgin) apprezza Luzzatti, su cui si esprime secondo i valori morali e populisti che già gli conosciamo: "...Luzzatti, vecchio apostolo della cooperazione, esperto finanziere e rinomato operatore sociale, appartenente alla vecchia formazione di destra del Parlamento”.

12. Sulla tragica vicenda del giovane V. V. Lebedincev, leggendaria figura del terrorismo social-rivoluzionario russo, cfr. il racconto di M. Osorgin, Neizvestnyj, po prozvišču Verner, nel periodico Na cužoj storone, Berlino-Praga, n.4, 1924, pp. 191- 203 (trad. italiana, Lo sconosciuto che si faceva chiamare Werner, in M. Osorgin, Un russo..., op. cit., pp. 127-151). Sul tema della sovrapposizione ideologica tra socialismo e anarchia, nel contesto dei rapporti italo-russi all'inizio del '900, cfr. A. Becca Pasquinelli, $M$. Osorgin, giornalista russo in Italia tra socialismo e anarchia (1908-1916), in Europa Orientalis, 1990, 1990, pp. 367-390. 
Al tema degli emigrati politici russi Osorgin non era nuovo, anzi, aveva già cominciato a lavorarci sopra da molto tempo: vediamo infatti un suo lungo articolo, intitolato "Emigranty o sebe", del 19 febbraio 1913, e un altro, sempre su RV, del 23 marzo, "Russkij kongress $v$ Rime" ${ }^{13}$ Nel primo di tali testi egli avverte di aver utilizzato il materiale ricavato dalle risposte a schede da lui distribuite già "due anni prima", cioè fin dal 1911, appunto a emigrati russi dalle più varie condizioni sociali, soprattutto per sondare in qual modo "l'emigrazione si rifletteva sui rappresentanti delle correnti del pensiero politico russo più radicale". Il lavoro si era annunciato troppo pesante per poterlo sostenere da solo, e Osorgin aveva dovuto lasciar perdere. Diventato il tema di grande attualità, lo riproponeva - riassunto, pubblicando varie risposte: l'esilio era per questi disadattati peggio di una prigione. Quindi, concludeva l'accorto Osorgin (due giorni prima dell'annuncio ufficiale dell'amnistia!) "la Russia deve spalancare le porte dell'enorme prigione, e spalancarle subito, prima che sia troppo tardi tanto per loro che per noi”. Nel secondo articolo qui citato, Osorgin rinnovava il proprio sostegno ai compatrioti, proponendo con energia il suo programma di ricupero, fondato sul tema della solidarietà sociale.

Forte della propria esperienza professionale, oltre che personale, sul complesso problema degli emigrati politici russi in Italia, il $28 \mathrm{feb}$ braio 1913 il nostro giornalista piomba dunque a Capri; tornerà due giorni dopo a Roma, dopo un casuale incontro con Gor'kij a spasso per l'isola e una lunga, memorabile sosta a casa sua. Ecco quel che egli annota a questo proposito il 3 marzo, al ritorno a Roma, in un suo diario "Vstreči": "Sono tornato ieri da Capri, dov'ero andato a trovare M. Gor'kij. Volevo controllare le voci che circolano su di lui in relazione all'amnistia (...) e 'intervistarlo' sulla questione degli emigrati amnistiati e di ritorno in Russia. Ieri sera l'ho incontrato per strada (stava tornando dal cinema di Capri, che frequenta abitualmente), e mi ha invitato a casa sua; era già tardi, quasi le dieci di sera". Gor'kij offre all'ospite una tazza di tè ( $v$ stolovoj), conversano insieme nel suo studio, quindi Osorgin viene invitato a pranzo per l'indomani: "E dopo pranzo - prosegue

13. Cfr. M. Osorgin, Gli emigranti a proposito di se stessi, RV, 19-2-1913, n. 41; Il congresso russo a Roma. Roma, 14 febbraio, RV, 23-3-1913, n. 69 ( trad. e commento in M. Osorgin, Un russo.., op. cit., pp119-125). Tamborra, op. cit., pp. 80-81. Per i titoli dei numerosi articoli dedicati da Osorgin ai problemi dei russi emigrati in Italia, cfr. M. Osorgin, Un russo..., op. cit., p. 125, n. 3, p. 10 per la sigla. 
il diario osorginiano - siamo stati a parlare per un paio d'ore, finché l'arrivo di I. Bunin non ha interrotto molto inopportunamente la nostra conversazione. Inopportunamente, è ovvio, per me" ${ }^{14}$

Quel pomeriggio stesso, tornato alla sua stanza d'albergo di Capri dopo una giornata tutta gor'kiana, egli lascia - da consegnare a Gor'kij - la breve lettera (n. 3), allegando un brano di conversazione già da lui composto, che avrebbe voluto inserire "se Voi me lo consentirete, in un articolo, finora, ovviamente non ancora scritto"; insomma dà carte blanche a Gor'kij di modificare o di cancellare ciò che non gli piacesse, o negarsi completamente, anche se, in questo caso "con mio gran dispiacere". Mentre sembra rimettersi completamente alla volontà del suo corrispondente, con l'ultima frase lo pone invece davanti ad una scelta: "Se invece va bene così, vogliate rimandarmi questi foglietti all'indirizzo romano dell'intestazione (per non disturbarvi a leggerli oggi stesso)". C'è tutto Osorgin, in questa sua volontà di chiarezza (senza se e senza ma) e qui coglieva il nocciolo del problema: infatti Gor'kij non era per niente deciso ad avvalersi - almeno per il momento - all'annuncio del "liberi tutti" proclamato dallo zar; ne aveva viste anche troppe, con il veto zarista all'Accademia, nel 1902, poi al nizloženie nel '09: sarebbe comunque tornato in Russia il 31 dicembre 1913, ostaggio ormai di un'utopia; infatti, fin dal febbraio 1913, come nota Bočarova (p. 391), "Gor'kij riceveva da Lenin una severa ammonizione (prjamoe nastavlenie) : "Spero che voi non riteniate che non si possa accettare l'amnistia?...".

A questo invito di Osorgin abbastanza pressante, Gor'kij risponde subito (lettera n. 4), il 3 marzo, in modo decisamente negativo, addobbando il suo rifiuto con eleganti, ma prudenti, imbarazzate reticenze: giusto - puntualizza - approfittare dell'amnistia, in quanto "dà modo all'uomo (cioè all'amnistiato) di prender coscienza del proprio valore culturale", ma egli non è d'accordo su ciò che segue nel testo osorgi-

14. Cfr. I. A. Bočarova, op. cit., pp. 389-390, cui "questo appunto è stato gentilmente presentato da Tat'jana Alekseevna Bakunina-Osorgina a Parigi nel 1989”. La curatrice annota inoltre (p.489) un'interessante testimonianza di questa visita improvvisa, ripresa dal diario di K. P. Pjatnickij, fedele amico di Gor'kij e curatore della sua rivista Znanie: "28 febbraio 1913. Vicino alla posta incontriamo Il'in. E' appena arrivato (...). Siamo andati al cinema, ma Gor'kij se n'era andato a casa. Il'in ha lasciato perdere (...). 2 marzo. Stamattina Il'in è partito. Era venuto sperando in un'intervista con Gor'kij. E' interessante sapere quel che scriverà”. 
niano presentato alla sua revisione - "ciascuno decide per se stesso" perché "in questo modo il motivo sociale viene sostituito dalle più svariate considerazioni soggettive": Gor'kij, in realtà, è molto indeciso. E il cono d'ombra, le scuse: "In verità, ho fondati motivi (l'ira di Lenin?) per restare nell'ombra; quanto al dibattito su questa questione, credetemi, questi motivi non sono di carattere personale, non sono affatto suscitati da alcun tipo di considerazioni sui miei comodi personali. E dunque, Michail Andreevič, anche se mi dispiace di rattristarvi, vorrei tuttavia che mi cancelliate dal vostro articolo e addirittura - vi prego - dimenticatemi”. Infine, c'è forse ancora una speranza di scelta? un esempio, uno strappo? "Lunačarskij, Agafonov e altri 'parigini' sembra abbiano deciso di non sfruttare i "doni dell'amnistia'". ${ }^{15}$ Alla luce del profondo interesse dimostrato da Osorgin con il suo lavoro sullo scottante tema della triste situazione dell'emigrazione russa in quegli anni, si comprende come, lungi dal desistere per il perentorio invito di Gor'kij a "farsi dimenticare", egli decida invece la linea dell'insistenza pertinace. Nella volontà di riallacciare il colloquio con Gor'kij, prototipo per lui dell'emigrato russo indeciso, per giunta suo amico, l'intelligent prezioso alla Russia di quell'ora, egli vede finalmente l'occasione di poter in qualche modo chiarire i dubbi e le disperazioni di tanti, aiutarli ad uscire da un'aporia straziante; il caso di Gor'kij, stretto fra uno zar infido e un "diabolico" Lenin, era però un difficile caso a sé.

E così, alla scoraggiante lettera di Gor'kij - una chiusura completa - Osorgin, come niente fosse, risponde col letterone inviato due giorni dopo, il 5 marzo (n.5). Più di una lettera, è piuttosto un lungo articolo, elaborato tuttavia non per un anonimo lettore, ma per colui che, nell'emigrazione russa del tempo, contava allora più di tutti. ${ }^{16}$ Osorgin imposta infatti il discorso come faceva nel suo lavoro giornalistico, se-

15. A. V. Lunačarskij, emigrato dopo il 1905, fu tra gli organizzatori della Scuola russa di Capri. tra il 1909 e il '15 visse in Francia e in Svizzera; tornò in Russia dopo la rivoluzione di febbraio. V. Agafonov (1863-1955), studioso di mineralogia, scrittore. Nel 1909 entrò tra i socialrivoluzionari. Dal 1921, emigrato, fu professore alla Sorbona.

16. Troviamo infatti qui le idee che Osorgin avrebbe ripreso in Russkie emigranty i 'russkij s'ezd', (Gli emigranti russi e il 'congresso di Roma') RV, luglio 1913, VII, pp. 283-299, dove, senza menzionare il nome di Gor'kij, egli così scriveva: "Recentemente mi è toccato avere sul tema 'nostalgia della patria' una conversazione, proseguita poi per corrispondenza, con un notissimo scrittore emigrato, che purtroppo non ho il diritto di nominare" (Cfr. Bočarova, p. 490). 
condo i cànoni di scuola social-populista, colorandolo però intanto della luce di una lusinghiera benevolenza: avrebbe sì voluto - dice pubblicare per prima l'intervista con Gorkij, tra quelle progettate con i più noti emigrati dell'intelligencija: al rifiuto oppostogli, si adegua, anzi, abolisce senz'altro tutto il progetto: "Se a Capri - prosegue Osorgin vi ho ascoltato come 'intervistatore', ora invece mi rivolgo a voi come emigrante, come un'unità della massa emigrata". Rimesso abilmente in questo modo il gioco, egli ricomincia la partita con un Gor'kij non più "mito", ma amico, compagno d'esilio. Dapprima vengono puntualmente illustrate le correnti di pensiero - quindi di comportamento - degli emigrati alle prese col problema dell'amnistia, per i quali si tratta di scrupoli morali piuttosto che utilitaristici, di scatti di orgoglio ribelle, di un groviglio inestricabile di emozioni, di esitazioni, di sentimenti contraddittori che paralizzano ogni iniziativa.

Il tono è quello di un'analisi sociologica condotta col metodo statistico, tipico dei maestri del pensiero critico populista - K. R. Michajlovskij, poi K. R. Kočarovskij, alla cui scuola egli si era formato. ${ }^{17}$ Statisticamente, per Osorgin le correnti di pensiero degli emigrati sono tre: alle prime due si è qui accennato, $\mathrm{e}$ "finalmente - scrive il gornalista è possibile anche una terza tendenza di pensiero", quella di coloro che, non resistendo alla "nostalgia della patria", decidono di tornare: uno stato d'animo che Gor'kij, da parte sua - aggiunge Osorgin - sembrava disconoscere completamente. Osorgin insiste invece su quest'ultima ragione "naturale" della nostalgia, la cui forza "dà più diritti che tutte le considerazioni sull'etica o sulla società", e prosegue la sua appassionata dissertazione, auspicando che ciascun emigrato decida per conto suo il proprio ritorno, senza farsi influenzare da pressioni altrui: "Dall'amnistia - scrive - sono escluso, e perciò provo una gran tranquillità". Con la propria esigenza di chiarezza, egli finisce col mettere in difficoltà il suo corrispondente, al quale proponeva una dichiarazione di scelta assolutamente personale, non condizionata da remore ideologiche o pres-

17. M. Osorgin, Po vostočnoj Riv'ere. Riv'era literaturnaja. Nervi, RV., n.38, 1914 (Sulla Riviera di Levante. La Riviera letteraria. Nervi, trad. A. Pasquinelli, Bollettino del CIRVI, Torino, n.8, luglio-dicembre 1983, p.254). L'autore ricorda l'attività svolta dalla comunità russa residente tra il 1906 e il 1908 a Sori, pittoresca località della riviera ligure di levante, nella "storica” villa Maria: “(...) Un piccolo padiglione era stato trasformato in studio di statistica, dove schioccavano i pallottolieri e funzionavano i regoli calcolatori”. Questo tema è ripreso da Osorgin anche in Lo sconosciuto... op., cit. 
sioni politiche. Alla fine della lettera, un'allusione cifrata a Bunin - ricordiamo l'appunto nel diario Vstreči sull'improvvisa visita caprese avrebbe determinato un ulteriore peggioramento dei rapporti epistolari tra i due esuli: l'ultima ipotesi rispetto all'esilio sarebbe stata valida per Gor'kij e per altri scrittori emigrati, ma - aggiunge maldestramente "oltre a grandi scrittori ce ne sono anche di piccoli (...). Purtroppo questo esempio non ho potuto presentarvelo, perché la nostra conversazione è stata interrotta dall'arrivo di ...(puntini di Osorgin), uno scrittore per il quale simili problemi non esistono".

Qualche giorno dopo (a metà marzo) ecco la risposta abbastanza irritata, ma puntuale, di Gor'kij (n. 6): "Permettetemi, Michail Andreevič, due o tre repliche alla vostra inattesa lettera (tono quasi confidenziale)". Gor'kij respinge l'accenno osorginiano al "cosmopolitismo" di certa intelligencija russa, al quale egli si sente estraneo, come contesta anche il fattore "nostalgia della patria", perché "la nostalgia per il luogo abituale è nota anche agli animali: ai cani, ai gatti" (SIC). Rifiuta inoltre risolutamente le indecisioni ascrittegli da Osorgin sul suo rientro in patria, e ribadisce che "non appena sarà necessario, partirò per la Russia". L'inatteso riferimento osorginiano a Bunin, amico fedelissimo e valido collaboratore letterario di Gor'kij, provocava nel famoso scrittore offeso un comprensibile moto d'ira: "(...) Protesto energicamente per la vostra valutazione delle opere e dell'importanza di I. A. Bunin - scusatemi, ma devo dirvi che tale valutazione vi degrada e che i puntini di sospensione da voi messi prima della parola "scrittore" sono veramente offensivi per me (...).

Osorgin risponde subito (n. 7), respingendo a sua volta - ironicamente - l'accezione del termine di nostalgia data da Gor'kij, nota anche agli animali: "A proposito - chiosa tra parentesi - ai cani è poco propria, ai gatti - sì". Perché mai - si domanda - i russi non dovrebbero provar nostalgia? "Io stesso - sostiene - sono russo, ma provo una nostalgia tale da non augurarla a nessun altro. (...) E non è vero che sia un sentimento basso, come non è basso il sentimento dell'amore per la propria madre, che è anch'esso di origine animale"; quindi si scusa per aver "inutilmente" attribuito a Gor'kij "incertezze e lacerazioni drammatiche". Ne respinge l'ira, riportando la propria gaffe su Bunin al doloroso tema dell'emigrazione, "...I puntini di sospensione non si riferiscono affatto a questa parola (...) Ho detto solo che, disponendo della possibilità di spostarsi liberamente, egli non manca di temi e di intrecci per le sue numerose opere (...). Ho chiamato questa una grande fortuna, della quale uno scrittore-emigrato è privo". 
Avverte un'incomprensione di fondo tra di loro, e ciò gli dispiace; conclude quindi amaramente con l'augurio che "i numerosi altri corrispondenti" di Gor'kij abbiano maggior fortuna di lui, e non ricevano risposte sgarbate come quella che gli era toccata. La franchezza reciproca dei due corrispondenti, la loro spietata sincerità, il loro (provvisorio ) distacco cementavano in realtà un'ininterrotta amicizia.

La corrispondenza italiana ritrovata termina qui: la prossima lettera nel volume - Osorgin a Gor'kij, 3 ottobre 1924 - verrà da Parigi, e Gor'kij gli risponderà, pochi giorni dopo, da Sorrento. L'ultima lettera della raccolta (n. 56) è di Gor'kij, scritta nel febbraio 1936, pochi mesi prima della tragica morte. 